\title{
Treatments of Peyronie's disease with Scutellaria baicalensis and surgery according to the disease course: a single-center retrospective study of 261 patients
}

\author{
Wen Ji Li"^, Jiewen Bao", Da-Chao Zheng, Jianhua Guo, Min-Kai Xie, Hui Ying Chen, Zhong Wang \\ Department of Urology and Andrology, Ninth People's Hospital, School of Medicine, Shanghai Jiaotong University, Shanghai, China \\ Contributions: (I) Conception and design: WJ Li, J Bao, HY Chen, Z Wang; (II) Administrative support: All authors; (III) Provision of study materials \\ or patients: WJ Li, HY Chen, Z Wang; (IV) Collection and assembly of data: WJ Li, J Bao, HY Chen, Z Wang; (V) Data analysis and interpretation: \\ All authors; (VI) Manuscript writing: All authors; (VII) Final approval of manuscript: All authors. \\ \#These authors contributed equally to this work. \\ Correspondence to: Zhong Wang. Professor, Department of Urology and Andrology, Ninth People's Hospital, School of Medicine, Shanghai Jiaotong \\ University, 639 Zhizaoju Road, Shanghai 200011, China. Email: zhongwang2010@sina.com; Hui Ying Chen. Associate Professor, Department of \\ Urology and Andrology, Ninth People's Hospital, School of Medicine, Shanghai Jiaotong University, 639 Zhizaoju Road, Shanghai 200011, China. \\ Email: newmailchy@163.com.
}

\begin{abstract}
Background: Oral medication therapies are more conventional than other non-surgical therapies in the acute phase of Peyronie's disease (PD). Although the commonly used oral drugs for PD have shown poor or indeterminate outcomes, most patients prefer oral medications. The aim of this study was to evaluate the efficacy and safety of Scutellaria baicalensis extract for treating acute-phase PD patients and examine the practicality of treatment strategies for PD according to the disease course.

Methods: This retrospective study was performed at our institution from 2005 to 2015 and analyzed the data of 261 patients with PD. The acute-phase PD patients received Scutellaria baicalensis extract for 6 months. After oral treatment, the patients with persistent curvature underwent surgical correction during the stable phase.

Results: During this study period, 183 patients received oral treatment with Scutellaria baicalensis, and 78 patients did not. Compared to the untreated patients, treatment with Scutellaria baicalensis had a significant effect in improving the symptoms of acute-phase PD. The mean time required for stabilization also showed a significant statistical difference. Treatment with Scutellaria baicalensis was safe and well-tolerated. After the disease stabilized, 70 and 31 patients with significant penile curvature underwent surgical correction by 16dot plication and great saphenous vein grafting procedures, respectively. At the one-year follow-up, complete penile straightening and penile length shortening were observed in $92.86 \%$ and $41.43 \%$ of the patients after 16 -dot plication and in $87.10 \%$ and $25.81 \%$ of the patients after grafting procedures, respectively. The postoperative Erectile Function domain of the International Index of Erectile Function scales were maintained in all patients after the 16 -dot plication procedure and decreased in $54.84 \%$ of the patients after the grafting procedure. Overall, $92.86 \%$ and $83.87 \%$ of the patients who received 16 -dot plication and grafting procedures, respectively, were satisfied with the final surgical results.

Conclusions: Treatment with extract of Scutellaria baicalensis seems to be beneficial for improvements in symptoms of acute phase PD and acceleration of the disease stabilization. The 16-dot plication and great saphenous vein grafting procedure seem effective options in the surgical management of the stable phase after Scutellaria baicalensis administration in the acute phase of PD.
\end{abstract}

Keywords: Grafting procedure; penile curvature; Peyronie's disease (PD); plication; Scutellaria baicalensis

$\wedge$ ORCID: 0000-0003-3689-5961. 
Submitted Dec 02, 2020. Accepted for publication Dec 31, 2020.

doi: 10.21037/apm-20-2389

View this article at: http://dx.doi.org/10.21037/apm-20-2389

\section{Introduction}

Peyronie's disease (PD) is recognized as a two-phase pathological condition of the penis, characterized by inelastic fibrous plaque formation in the tunica albuginea (1). Usually, the natural history of $\mathrm{PD}$ is progressive, and spontaneous regression is rare (2).

Many treatment approaches and their clinical outcomes have been described in the literature, including oral, intralesional, mechanical, topical, and surgical therapies $(3,4)$. Several oral medications have been used for the treatment of PD based on their antioxidant and anti-fibrotic effects $(3,4)$. However, the routine clinical use of oral agents for PD such as vitamin E, tamoxifen, or omega-3 fatty acids has shown poor or indeterminate outcomes. Therefore, these treatments are not supported by the American Urological Association guidelines or the 2010 International Consultation on Sexual Medicine (5). Even so, oral therapies are used more than other non-surgical therapies during the acute phase of $\mathrm{PD}$, and most patients prefer oral medications in clinical practice. Therefore, it is necessary to explore a new oral pharmacotherapy for treating the acute phase of PD.

Previously, we found that monocyte chemoattractant protein 1 (MCP-1), a profibrotic factor, was expressed at higher levels in cells from plaques ( $\mathrm{P}$ cells) in the tunica albuginea of PD patients $(6,7)$. In another study, we found that wogonin, a flavonoid extracted from the root of Scutellaria baicalensis, significantly inhibited cell proliferation and downregulated MCP-1 expression in $\mathrm{P}$ cells, indicating that wogonin may be a worthy candidate for preclinical trials in men with PD (8). In clinical practice, we administered extract of Scutellaria baicalensis to treating acute phase of PD patients. After treatment with Scutellaria baicalensis, if the patients still had persistent penile curvature severely precluded intercourse, the patients underwent surgical correction during the stable phase. The aim of this study was to evaluate the therapeutic effect of different treatment strategies in PD according to the course of the disease, including evaluation of the efficacy and safety of Scutellaria baicalensis extract in treating acute-phase PD patients and the outcomes of surgical correction of persistent penile curvature during the stable-phase PD patients.
We present the following article in accordance with the STROBE reporting checklist (available at http://dx.doi. org/10.21037/apm-20-2389).

\section{Methods}

This retrospective study was approved by the institutional research ethics committee of the Ninth People's Hospital, School of Medicine, Shanghai Jiaotong University (No. SH9H-2019-T52-2). Since only a medical record review was performed, this study was exempt from informed consent. This study was conducted in accordance with the declaration of Helsinki (as revised in 2013). Following approval from the Institutional Ethics Review Board, we retrospectively reviewed the charts of patients with $\mathrm{PD}$ at a single center from 2005 to 2015 . The inclusion criteria were (I) patients with acute-phase $\mathrm{PD}$ which defined as have any of the symptoms of penile plaques, erectile pain, or penile curvature in the previous 6 months; (II) acute-phase PD patients who received oral treatment with Scutellaria baicalensis extract and were followed until 6 months after the disease stabilized; (III) acute-phase PD patients who consented to be followed until 6 months after the disease stabilized although they refused oral treatment with Scutellaria baicalensis; (IV) patients who still had significant penile curvature (greater than 30 degrees) severely precluded intercourse after treatment with Scutellaria baicalensis were underwent surgical correction after the disease stabled for at least 6 months; (V) Among the patients who did not receive Scutellaria baicalensis treatment, the patients who had significant penile curvature (greater than 30 degrees) severely precluded intercourse were underwent surgical correction after the disease stabled for at least 6 months; and (VI) patients followed for at least one year after surgical correction, whether they were treated with Scutellaria baicalensis or not. The exclusion criteria were (I) among the patients treated with Scutellaria baicalensis, who received any treatment for PD before baseline assessment or received other treatments for PD concurrently during the followup period; (II) among the patients did not receive Scutellaria baicalensis treatment, who received any treatment for $\mathrm{PD}$ before the baseline assessment or received any treatment for PD during the follow-up period; and (III) patients who had 
erectile dysfunction (ED) prior to the onset of PD.

The variables analyzed included patient demographics and disease characteristics. The duration of disease was determined by differences between the date at the examination and the date of onset of the first symptom. Penile pain during erection was assessed using a numerical rating scale (NRS). The angle and degree of penile curvature during maximum erection were documented by auto-photography using the Kelami technique (9). If a photograph could not be obtained, an artificial erection was induced by intra-cavernosal injection of papaverine $(30 \mathrm{mg})$. The International Index of Erectile Function (IIEF) erectile function domain (EF) score was used to evaluate erectile function, and a score below 26 identified ED (10). Color Doppler ultrasound was used to measure the three dimensions of the plaque (length, width, and thickness) by an experienced sonographer, and the plaque volume was determined $\left(\mathrm{cm}^{3}\right)$ using the prolate ellipsoid formula (11). The time required for stabilization was determined by the difference between the dates the patients had no painful erections and further progressive deformity or curvature for at least 3 months and the date of onset of the first symptom.

The acute-phase PD patients received Scutellaria baicalensis extract, which was prepared by boiling $12 \mathrm{~g}$ of the dried root of the plant in $600 \mathrm{~mL}$ water until only $300 \mathrm{~mL}$ of the solution remained. An oral administration of $150 \mathrm{~mL}$ of this solution was given each morning and evening for 6 months. To evaluate the safety of Scutellaria baicalensis, regular monitoring of adverse events was performed 1 and 3 months after treatment and at the end of treatment. The adverse events were classified as no or mild, moderate, or severe based on clinical scores. If the patient still had persistent significant curvature (greater than 30 degrees) after oral treatment, surgical management including the 16dot technique and great saphenous vein grafting procedure were performed in patients who had stable disease for at least 6 months. Patients with persistent significant curvature among patients who refused Scutellaria baicalensis treatment also underwent the same surgical management. Before surgery, all patients underwent measurement of flaccid stretched penile length (SPL) dorsally from pubis to meatus under maximal extension of the phallus and compressing suprapubic fat pad to the pubic bone. The degree of penile curvature was also documented by using the Kelami technique (9). Although penile prosthesis (PP) implantation was recommended for those patients with ED who did not respond to phosphodiesterase type 5 inhibitors (PED5is), the patients refused PP implantation because they were older and had a low sexual desire or because of economic reasons.

The indications for the 16-dot plication procedure in the present study were patients who had adequate erectile rigidity for intercourse with or without pharmacotherapy, an angle of penile curvature of less than $60^{\circ}$ and without an indentation or hourglass deformity, a stretched length of more than $10 \mathrm{~cm}$, and an anticipated loss of length less than $20 \%$ of the erect or stretched length (12). The surgical procedure was performed according to the technique described by Lue (13). The indications for the great saphenous vein grafting procedure in the present study were patients who had adequate erectile rigidity for intercourse with or without pharmacotherapy, more severe curves greater than $60^{\circ}$, a complex deformity, an indentation or hourglass deformity, a stretched length less than $10 \mathrm{~cm}$, and an anticipated loss of length of more than 20\% (12). The surgical procedure was performed according to the technique described by Lue (14).

After surgery, the patients were recommended to abstain from all forms of sexual intercourse for 2 months. Although penile traction therapy or vacuum devices were not used, the patients were recommended to take daily low dose PED5is two weeks after surgery and continue for 2 months to promote nocturnal erections and prevent graft contracture. At follow-up, erectile function was assessed using the IIEF-EF questionnaire at 3 and 6 months and one year postoperatively. The SPL and residual curvature were also measured in the same way as before operation at 3 and 6 months and 1 year postoperatively. To assess overall satisfaction, the patients were asked whether they were (I) very satisfied, (II) somewhat satisfied, or (III) not at all satisfied with the surgery and the subsequent results at 3 and 6 months and one year postoperatively.

\section{Statistical analysis}

All statistical analyses were performed using SPSS 20.0 software (SPSS Inc., Chicago, IL, USA). The baseline and follow-up continuous variables are presented as mean \pm standard deviation (SD) for normal distributions and as median (interquartile range, IQR) for non-normal distributions. To compare the demographic characteristics and therapeutic outcomes, we used Student's $t$-test for normally distributed data. Otherwise, the Mann-Whitney U-test was performed. The baseline and follow-up categorical variables are presented as $\mathrm{n}(\%)$ and tested using the chi-squared $\left(\chi^{2}\right)$ test. A $\mathrm{P}$ value of $<0.05$ was considered 
statistically significant.

\section{Results}

\section{Evaluation of the therapeutic efficacy and safety of Scutellaria baicalensis extract}

During this study period, 261 acute-phase PD patients met the inclusion criteria and the age varied between 26 and 69 years. Among them, 210 (80.46\%) and 226 (86.59\%) patients presented with penile pain on erection and palpable penile plaques, respectively. Penile curvature was presented in $161(61.69 \%)$ cases and ED was observed in 92 (35.25\%) patients. Among them, 183 patients received oral treatment with Scutellaria baicalensis extract and 78 patients did not. Statistical comparison of the baseline demographics and disease characteristics in the two groups did not reveal any significant differences (Tables 1,2).

In the patients treated with Scutellaria baicalensis extract, disease stabilized at an average of 12.7 months, whereas further progression was stopped at an average of 18.01 months in the untreated patients (Table 1). Statistical analysis showed that treatment with Scutellaria baicalensis extract had some benefits for improving erectile pain, plaque volume, penile curvature, and erectile function (Tables 3-6).

After 6 months of Scutellaria baicalensis treatment, penile pain resolved or decreased in $16.89 \%$ and $69.59 \%$ of the patients, respectively. New onset of penile pain presented in only $11.43 \%$ of the Scutellaria baicalensis-treated patients. In the same period, none of the untreated patients had penile pain resolution. In contrast, $88.71 \%$ of the patients had increased erectile pain. Furthermore, the new onset of penile pain presented in $56.25 \%$ of the untreated patients (Table 3).

After 6 months of Scutellaria baicalensis treatment, the proportion of patients with reduced plaque volume and curvature was significantly higher than that of the untreated patients (43.4\% and $40.0 \%$ vs. $26.87 \%$ and $13.04 \%)$. In addition, the proportion of new-onset of penile curvature in patients without prior curvature and the curvature degree was significantly higher in untreated patients than in Scutellaria baicalensis-treated patients (Tables 4,5). At the stable stage of the disease, the proportion of patients requiring surgical correction in the untreated group was significantly higher than that in the Scutellaria baicalensistreated patients (57.69\% vs. 32.79\%) (Tables 4,5).

After 6 months of treatment, regular erections were recovered in $29.23 \%$ of the patients, whereas no recovery occurred in the untreated patients. In addition, no onset of ED occurred in patients treated with Scutellaria baicalensis who had no problems of rigidity at the first visit, whereas $35.29 \%$ of the untreated patients had ED in the stable phase who did not present with it initially (Table 6).

After the administration of Scutellaria baicalensis, 146 adverse events occurred, and no serious adverse events were observed. All adverse events were rated as mild and resolved without further treatment. None of the patients discontinued treatment because of an adverse event (Table 7).

\section{Evaluation of the therapeutic efficacy of surgery after Scutellaria baicalensis treatment}

At least 6 months after the disease stabilized, 60 (32.79\%) and $45(57.69 \%)$ patients treated with or not treated with Scutellaria baicalensis extracts, respectively, required surgical correction of penile curvatures. Among them, 70 and 31 patients underwent surgical correction by $16-$ dot plication and grafting procedures, respectively (Table 8). The remaining four patients with severe ED who did not respond to PED5is refused the recommended PP implantation.

At the 1-year follow-up, complete penile straightening was observed in $92.86 \%$ and $87.1 \%$ of the patients after 16-dot plication and grafting procedures, respectively. Although slight residual curvature (less than 20 degrees) was observed in the remaining cases, the patients did not receive further treatment because it did not affect sexual intercourse (Table 8).

Shortening of penile length occurred in $41.43 \%$ and $25.81 \%$ of the patients, and the range of loss was $0.5-2.6 \mathrm{~cm}$ and $0.2-1.2 \mathrm{~cm}$ after $16-$ dot plication and grafting procedures, respectively. Among these patients, five complained of a shortened penile length although sexual intercourse was not affected (Table 8).

Postoperative IIEF-EF scores were maintained in all patients who received the 16-dot plication procedure and slightly improved in $48.57 \%$ patients. Moreover, patients who had difficulty with intercourse because of penile curvature seemed to attain benefit in sexual activity after curvature correction while $54.84 \%$ of the patients who received the venous grafting procedure reported varying degrees of decreased erectile rigidity. However, all these patients achieved adequate erectile capacity after receiving PED5is and were able to have successful sexual intercourse (Table 8).

There was no postoperative curvature recurrence in any 
Table 1 Clinical and demographic features of PD patients

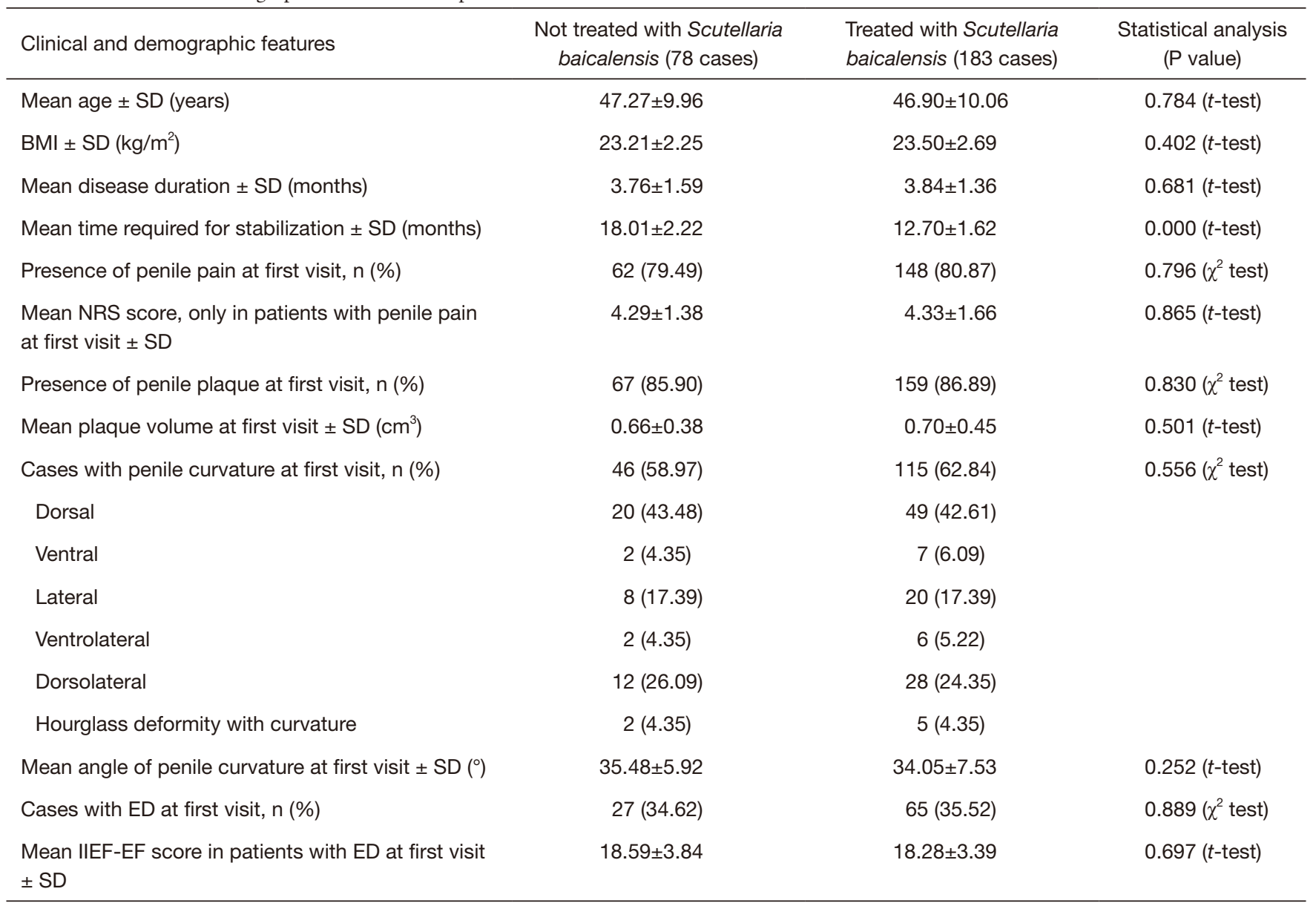

ED, erectile dysfunction; IIEF-EF, International Index of Erectile Function (IIEF) erectile function domain (EF); SD, standard deviation.

patients treated with Scutellaria baicalensis. In patients not treated with Scutellaria baicalensis, curvature recurrence did not occur in the original area. However, the new onset of palpable plaques and curvature occurred in two (16.67\%) patients at 12 -month follow-up at sites distal or proximal to the location of the original penile curvature (Table 8).

Complications such as diminished postoperative penile sensation, palpable suture knots, and painful erection were noticed in $11.43 \%, 22.86 \%$, and $5.71 \%$ of the patients who received 16-dot plication, and in $22.58 \%, 12.9 \%$, and $6.45 \%$ of the patients who received grafting procedures, respectively. However, these complications did not affect sexual intercourse. Other surgery-related complications including penile hematoma, glandular ischemia, and wound infection were not observed in any patient (Table 9). Overall, $92.86 \%$ and $83.87 \%$ of the patients who received 16 -dot plication and grafting procedures were satisfied with the final surgical results (Table 8).

\section{Discussion}

Scutellaria baicalensis is a widely used traditional Chinese herbal medicine and the roots of Scutellaria baicalensis contain more than 40 kinds of flavonoids. Among them, baicalin, baicalein, wogonin, and oroxylin A are the main active components (15). Several in vitro and in vivo studies have demonstrated that a wide range of Scutellaria baicalensis extract concentrations had excellent anti-fibrotic activity in kidney fibroblasts $(16)$, renal fibrosis $(17,18)$, myocardial fibrosis (19), hepatic fibrosis (20), and fibrotic lung disease (21). PD is a well-established fibrotic disease, and several anti-fibrotic therapies have been described for 
Table 2 Concurrent risk factors

\begin{tabular}{|c|c|c|c|}
\hline Concurrent risk factors & $\begin{array}{l}\text { Not treated with Scutellaria } \\
\text { baicalensis ( } 78 \text { cases) }\end{array}$ & $\begin{array}{l}\text { Treated with Scutellaria } \\
\text { baicalensis (183 cases) }\end{array}$ & $\begin{array}{c}\text { Statistical analysis } \\
(\mathrm{P} \text { value })\end{array}$ \\
\hline Coronary artery disease, $\mathrm{n}(\%)$ & $6(7.69)$ & $14(7.65)$ & 0.991 ( $\chi^{2}$ test $)$ \\
\hline Diabetes mellitus, n (\%) & $7(8.97)$ & $16(8.74)$ & 0.952 ( $\chi^{2}$ test) \\
\hline Hypertension, n (\%) & $18(23.08)$ & $42(22.95)$ & $0.982\left(\chi^{2}\right.$ test $)$ \\
\hline Penile trauma, n (\%) & $5(6.41)$ & $11(6.01)$ & 0.902 ( $\chi^{2}$ test $)$ \\
\hline Dupuytren's contracture, n (\%) & $1(1.28)$ & $2(1.09)$ & 0.896 ( $\chi^{2}$ test $)$ \\
\hline Cigarette smoking, n (\%) & $20(25.64)$ & $46(25.14)$ & $0.932\left(\chi^{2}\right.$ test $)$ \\
\hline
\end{tabular}

Table 3 Changes of erectile pain after treatment with Scutellaria baicalensis

\begin{tabular}{|c|c|c|c|}
\hline Outcomes & $\begin{array}{l}\text { Not treated with Scutellaria } \\
\text { baicalensis ( } 78 \text { cases) }\end{array}$ & $\begin{array}{l}\text { Treated with Scutellaria baicalensis } \\
\text { (183 cases) }\end{array}$ & $\begin{array}{l}\text { Statistical analysis } \\
\text { ( } P \text { value })\end{array}$ \\
\hline Mean pain resolution, $\mathrm{n} / \mathrm{N}(\%)$ & 0 & $25 / 148(16.89)$ & 0.001 ( $\chi^{2}$ test $)$ \\
\hline Mean pain decrease \pm SD & $-2.25 \pm 0.50$ & $-2.00 \pm 0.94$ & 0.599 (t-test) \\
\hline Mean pain increase, $\mathrm{n} / \mathrm{N}(\%)$ & $55 / 62(88.71)$ & $14 / 148(9.46)$ & 0.000 ( $\chi^{2}$ test $)$ \\
\hline $\begin{array}{l}\text { Mean onset of penile pain in } \\
\text { patients with no pain, n/N (\%) }\end{array}$ & $9 / 16(56.25)$ & 4/35 (11.43) & 0.001 ( $\chi^{2}$ test $)$ \\
\hline Mean onset of penile pain \pm SD & $5.33 \pm 1.23$ & $1.50 \pm 0.58$ & 0.000 (t-test) \\
\hline
\end{tabular}

$\mathrm{SD}$, standard deviation.

Table 4 Changes of plaque after treatment with Scutellaria baicalensis

\begin{tabular}{lccc}
\hline Outcomes & $\begin{array}{c}\text { Not treated with Scutellaria } \\
\text { baicalensis }(78 \text { cases })\end{array}$ & $\begin{array}{c}\text { Treated with Scutellaria baicalensis } \\
(183 \text { cases })\end{array}$ & $\begin{array}{c}\text { Statistical analysis } \\
(P \text { value })\end{array}$ \\
\hline Mean plaque decrease, $\mathrm{n} / \mathrm{N}(\%)$ & $18 / 67(26.87)$ & $69 / 159(43.40)$ & $0.020\left(\chi^{2}\right.$ test $)$ \\
Mean plaque decrease $\pm \mathrm{SD}\left(\mathrm{cm}^{3}\right)$ & $-0.44 \pm 0.31$ & $-0.52 \pm 0.33$ & $0.384(t$-test $)$ \\
Mean plaque increase $\pm \mathrm{SD}\left(\mathrm{cm}^{3}\right)$ & $0.70 \pm 0.72$ & $0.50 \pm 0.60$ & $0.090(t$-test $)$ \\
$\begin{array}{l}\text { Mean onset of plaque in patients } \\
\text { with no plaque, } \mathrm{n} / \mathrm{N}(\%)\end{array}$ & $11 / 11(100.0)$ & $24 / 24(100.0)$ & $1.000\left(\chi^{2}\right.$ test $)$ \\
Mean onset of plaque $\pm \mathrm{SD}\left(\mathrm{cm}^{3}\right)$ & $0.54 \pm 0.43$ & $0.37 \pm 0.22$ & $0.141(t$-test $)$ \\
\hline
\end{tabular}

$\mathrm{SD}$, standard deviation.

PD, including potassium para-aminobenzoate (POTABA), tamoxifen, carnitine, pentoxifylline, and interferon $\alpha-2 b$ by oral or intralesional injection, with various outcomes reported (22).
In the present study, a raw extract of Scutellaria baicalensis was used for treating acute-phase PD because of its antifibrotic effect, which was demonstrated in our previous study (8). As a result, a significantly increased proportion 
Table 5 Changes of penile curvature after treatment with Scutellaria baicalensis

\begin{tabular}{|c|c|c|c|}
\hline Outcomes & $\begin{array}{l}\text { Not treated with Scutellaria } \\
\text { baicalensis ( } 78 \text { cases) }\end{array}$ & $\begin{array}{l}\text { Treated with Scutellaria } \\
\text { baicalensis (183 cases) }\end{array}$ & $\begin{array}{l}\text { Statistical analysis } \\
(P \text { value })\end{array}$ \\
\hline Mean penile curvature resolution, n/N (\%) & 0 & 2/115 (1.74) & 0.368 ( $\chi^{2}$ test $)$ \\
\hline Mean penile curvature increase, $\mathrm{n} / \mathrm{N}(\%)$ & $31 / 46(67.39)$ & $56 / 115(48.70)$ & 0.032 ( $\chi^{2}$ test) \\
\hline Mean penile curvature reduction $\pm \mathrm{SD}\left({ }^{\circ}\right)$ & $15.50 \pm 5.13$ & $16.52 \pm 4.67$ & 0.62 (t-test) \\
\hline $\begin{array}{l}\text { Mean onset of penile curvature in patients } \\
\text { with no curvature, } n / N(\%)\end{array}$ & $27 / 32(84.38)$ & $36 / 68(52.94)$ & $0.002\left(\chi^{2}\right.$ test $)$ \\
\hline Mean onset of penile curvature \pm SD & $33.52 \pm 14.48$ & $24.14 \pm 7.79$ & 0.002 (t-test) \\
\hline
\end{tabular}

SD, standard deviation.

Table 6 Mean change in erectile function at stable phase

\begin{tabular}{|c|c|c|c|}
\hline Outcomes & $\begin{array}{l}\text { Not treated with Scutellaria } \\
\text { baicalensis ( } 78 \text { cases) }\end{array}$ & $\begin{array}{l}\text { Treated with Scutellaria } \\
\text { baicalensis ( } 183 \text { cases) }\end{array}$ & $\begin{array}{l}\text { Statistical analysis } \\
\text { ( } P \text { value })\end{array}$ \\
\hline $\begin{array}{l}\text { Mean improvement in penile rigidity in } \\
\text { patients with } E D, n / N(\%)\end{array}$ & $0 / 27(0)$ & $46 / 65(70.77)$ & 0.000 ( $\chi^{2}$ test $)$ \\
\hline $\begin{array}{l}\text { Mean recovery of penile rigidity in patients } \\
\text { with } E D, n / N(\%)\end{array}$ & $0 / 27(0)$ & $19 / 65(29.23)$ & $0.002\left(\chi^{2}\right.$ test $)$ \\
\hline $\begin{array}{l}\text { Onset of ED in patients with previously normal } \\
\text { penile rigidity, } n / N(\%)\end{array}$ & $18 / 51$ (35.29) & 0/118 (0) & $0.000\left(\chi^{2}\right.$ test $)$ \\
\hline
\end{tabular}

of patients with reduced plaque volume and decreased penile pain during erection and penile curvature was observed in patients treated with Scutellaria baicalensis compared to the untreated patients. After treatment with Scutellaria baicalensis, IIEF scores also significantly increased and no onset of ED occurred. Furthermore, the disease stabilized 12.7 months after the onset of symptoms in the patients treated with Scutellaria baicalensis, whereas the disease progression did not stop until 18.01 months in the untreated patients. These results indicate that Scutellaria baicalensis extract had a benefit in the treatment of acutephase PD. Although some studies demonstrated that Scutellaria baicalensis had potential toxicity, it has been widely regarded as a safe and nontoxic traditional Chinese medicine in China for thousands of years (15). Preclinical safety data from laboratory assessments also showed that Scutellaria baicalensis was safe and well-tolerated by healthy subjects and showed no signs of liver or kidney toxicity (23). In our study, except for transient and mild adverse events, no serious adverse events were observed and none of the patients discontinued treatment because of an adverse event. The favorable safety profile warrants further clinical studies of Scutellaria baicalensis for PD treatment.

However, oral treatments appear to be moderately efficacious for symptomatic men in the acute phase of PD or in men in the chronic phase of PD who are not ready to consider surgical therapy. If conservative therapies have failed and the patient is significantly bothered by penile 
Table 7 Summary of Scutellaria baicalensis treatment-related adverse events

\begin{tabular}{lcc}
\hline Adverse events & $\begin{array}{c}\text { No. (\%) of subjects } \\
(183 \text { cases })\end{array}$ & Grade \\
\hline Abdominal pain & $15(8.20)$ & Mild \\
Abdominal distention & $14(7.65)$ & Mild \\
Constipation & $11(6.01)$ & Mild \\
Diarrhea & $16(8.74)$ & Mild \\
Nausea & $24(13.11)$ & Mild \\
Dry mouth & $22(12.02)$ & Mild \\
Erythema & $11(6.01)$ & Mild \\
Itchy skin & $13(7.10)$ & Mild \\
$\begin{array}{l}\text { Blood pressure increase or } \\
\text { decrease }\end{array}$ & $0(0.00)$ & Mild \\
Anemia & $2(1.09)$ & Mild \\
Blood leukocyte decrease & $2(1.09)$ & Mild \\
Proteinuria & $5(2.73)$ & Mild \\
Alanine transaminase & $5(2.73)$ & Mild \\
increase & $6(3.28)$ & Mild \\
Aspartate aminotransferase & & \\
increase & &
\end{tabular}

deformity, surgical correction can be considered (4). In the present study, $32.79 \%$ of the patients treated with Scutellaria baicalensis and $57.69 \%$ of the untreated patients still had persistent penile curvature at least 6 months after the disease stabilized. Intralesional injection therapy with collagenase Clostridium histolyticum $(\mathrm{CCH})$ was shown to be efficacious and less invasive than surgery $(24,25)$. However, surgical correction remains the gold standard treatment option for patients in the stable phase of PD (26). In addition, $\mathrm{CCH}$ was not commercially available in our country during the treatment of those patients. Therefore, surgical correction was recommended for those patients in our clinical practice.

Beginning with the Nesbit procedure, several techniques of plication have been offered to PD patients with various outcomes. The 16-dot plication technique is a minimally invasive procedure, which exhibited excellent results in the surgical management of patients with stable PD (13). Incision or partial plaque excision and grafting techniques are recommended for patients with adequate rigidity, more severe curves of greater than $60-70^{\circ}$, a complex deformity, or narrowing with an hourglass or hinge effect (12). Many different types of autograft, allograft, xenograft, or synthetic materials have been used in grafting surgical

Table 8 Surgical outcomes at the one-year follow-up

\begin{tabular}{|c|c|c|}
\hline Surgical outcomes & 16-dot plication procedure & Grafting procedure \\
\hline Cases not treated with Scutellaria baicalensis ( $\mathrm{n})$ & 33 & 12 \\
\hline Complete straightening, $\mathrm{n} / \mathrm{N}(\%)$ & $65 / 70(92.86)$ & $27 / 31(87.10)$ \\
\hline Slight residual curvature (less than 20 degrees), n/N (\%) & $5 / 70(7.14)$ & $4 / 31(12.90)$ \\
\hline Onset of shortening, $<1.0 \mathrm{~cm}, \mathrm{n} / \mathrm{N}(\%)$ & $9 / 70(12.86)$ & $7 / 31(22.58)$ \\
\hline Onset of shortening, $1.0-2.0 \mathrm{~cm}, \mathrm{n} / \mathrm{N}(\%)$ & $15 / 70(21.43)$ & $1 / 31(3.23)$ \\
\hline Onset of shortening, $>2.0 \mathrm{~cm}, \mathrm{n} / \mathrm{N}(\%)$ & $5 / 70(7.14)$ & 0 \\
\hline Mean shortening, $\mathrm{cm} \pm \mathrm{SD}$ & $1.37 \pm 0.55$ & $0.53 \pm 0.30$ \\
\hline Mean changes in IIEF-EF score in patients with ED at first visit \pm SD & $1.18 \pm 0.39$ & $-2.06 \pm 0.66$ \\
\hline Onset of new palpable plaque and curvature & $0(0)$ & 2/12 (16.67) \\
\hline Satisfaction with surgical outcomes [somewhat or very satisfied, n/N (\%)] & $65 / 70(92.86)$ & 26/31 (83.87) \\
\hline
\end{tabular}

$\mathrm{ED}$, erectile dysfunction; IIEF-EF, International Index of Erectile Function (IIEF) erectile function domain (EF); SD, standard deviation. 
Table 9 Surgical complications

\begin{tabular}{lcc}
\hline Complications & 16-dot plication procedure & Grafting procedure \\
\hline Diminished penile sensation, n/N (\%) & $8 / 70(11.43)$ & $7 / 31(22.58)$ \\
Palpable suture knots, n/N (\%) & $16 / 70(22.86)$ & $4 / 31(12.9)$ \\
Painful erection, n/N (\%) & $4 / 70(5.71)$ & $2 / 31(6.45)$ \\
Penile hematoma (\%) & 0 & 0 \\
Glandular ischemia (\%) & 0 & 0 \\
Wound infection (\%) & 0 & 0
\end{tabular}

procedures with various outcomes $(27,28)$. Venous patch grafts for PD have gained wide popularity because of their physiological characteristics with no risk of rejection or shrinkage. In addition, the endothelial coating of the vein can easily contact cavernosal tissue and regain blood supply $(29,30)$. The most frequently reported complications of surgical corrections are incomplete straightening, curvature recurrence, penile shortening, and ED either using plication procedures or grafting techniques (12). In the present study, at the one-year follow-up, complete penile straightening was observed in $92.86 \%$ and $87.10 \%$ of the patients after 16-dot plication and grafting procedures, respectively. However, the remaining patients complained of slight residual curvature but without an effect on sexual intercourse. Interestingly, the present study showed that neither postoperative palpable penile plaques nor curvature recurred in any patient treated with Scutellaria baicalensis extract or in the original area of the untreated patients, whereas palpable plaques and curvatures recurred at sites distal or proximal to the original penile curvature site in the untreated patients. These results showed that even though one lesion site is stable, new lesions may occur in other areas in PD. Therefore, prior to any treatment for PD, patients should be counseled on the possibility of palpable penile plaques and curvature occurrence in new areas. These results also indicated that the use of Scutellaria baicalensis extract had a benefit for preventing penile plaques and curvature occurrence.

One of the consequences of PD is penile shortening that can cause significant personal distress. An additional loss of penile length may occur after surgical management and that is a great concern for the patient. Previous studies have demonstrated that penile shortening occurred in $41-100 \%$ and $0-40 \%$ of the patients following various plication procedures and grafting techniques, respectively $(22,28,31,32)$. In our study, SPL loss occurred in $41.43 \%$ and $25.81 \%$ of the patients following 16 -dot plication and grafting techniques with a mean loss of 1.37 and $0.53 \mathrm{~cm}$, respectively. Although five patients complained of shortened penile length following 16-dot plication, none of the patients stated that sexual intercourse was adversely affected by the loss of penile length. Even though penile shortening rarely results in intercourse difficulty (33), patients often perceive that the loss of length is greater than the actual loss (34). Therefore, this side effect must be clearly explained to the patients prior to the surgery, and measuring and documenting the penile length preoperatively is recommended, regardless of what technique is used $(31,34)$.

According to the literature, the plication procedure usually does not affect erectile function $(13,35)$, whereas the postoperative ED rate after surgery with a venous patch graft ranged between 0 and $50 \%$ (27). In the present study, postoperative sexual function was maintained in patients who underwent the 16-dot plication procedure. Moreover, patients who had difficulty with intercourse because of penile curvature seemed to attain a benefit in sexual activity after curvature correction, whereas $54.84 \%$ of the patients who received a venous grafting surgical procedure reported varying degrees of decreased erectile rigidity. Therefore, the preoperative evaluation of erectile capacity is critical for choosing the surgical technique.

This study had several important limitations, such as the retrospective design, which includes inherent bias. In addition, a raw extract of Scutellaria baicalensis was used for treating acute-phase PD in the present study. Since the components of herbal extracts are complex, multiple constituents may have different effects, which may confound the exact role of Scutellaria baicalensis in treating acute-phase PD. This is one of the important limitations of this study. Further studies are needed to clarify the exact role of Scutellaria baicalensis by isolating an active fraction from Scutellaria baicalensis. Finally, only 
the IIEF-EF questionnaire, a subjective tool, was used to evaluate erectile function in the present study. The IIEFEF questionnaire is an applicable and amenable tool for the evaluation of erectile function, which may be influenced by some pre-and postoperative factors such as depression after clinical diagnosis, the surgical decision, stress, pain at the surgical site, and the resulting compromise of libido. Therefore, objective assessments such as the nocturnal penile tumescence test, video-provoked erectile response measurements, or penile Doppler ultrasound may provide more data on erectile function.

\section{Conclusions}

The present study showed that treatment with Scutellaria baicalensis extract seemed to be beneficial in improving penile plaque volume, erectile pain, penile curvature, and erectile function in patients with acute-phase PD. Early treatment with Scutellaria baicalensis extract also accelerated the stabilization of the disease and reduced the possibility of recurrence. The 16-dot plication and great saphenous vein grafting procedures seem effective options in the surgical management of the stable phase after Scutellaria baicalensis administration in the acute phase of PD. Although our study achieved significant results, further randomized controlled trials are necessary to clarify the exact role of Scutellaria baicalensis extract in the treatment of PD.

\section{Acknowledgments}

Funding: This work was supported by grants from the National Natural Science Foundation of China (Grant No. 81700582), Shanghai Jiaotong University School of Medicine Multi-center Clinical Research Project (DLY201809) and Program for outstanding academic leader of Huangpu District.

\section{Footnote}

Reporting Checklist: The authors have completed the STROBE reporting checklist. Available at http://dx.doi. org/10.21037/apm-20-2389

Data Sharing Statement: Available at http://dx.doi. org/10.21037/apm-20-2389

Conflicts of Interest: All authors have completed the ICMJE uniform disclosure form (available at http://dx.doi. org/10.21037/apm-20-2389). The authors have no conflicts of interest to declare.

Ethical Statement: The authors are accountable for all aspects of the work in ensuring that questions related to the accuracy or integrity of any part of the work are appropriately investigated and resolved. This retrospective study was approved by the institutional research ethics committee of the Ninth People's Hospital, School of Medicine, Shanghai Jiaotong University (No. SH9H2019-T52-2). Since only a medical record review was performed, this study was exempt from informed consent. This study was conducted in accordance with the declaration of Helsinki (as revised in 2013).

Open Access Statement: This is an Open Access article distributed in accordance with the Creative Commons Attribution-NonCommercial-NoDerivs 4.0 International License (CC BY-NC-ND 4.0), which permits the noncommercial replication and distribution of the article with the strict proviso that no changes or edits are made and the original work is properly cited (including links to both the formal publication through the relevant DOI and the license). See: https://creativecommons.org/licenses/by-nc-nd/4.0/.

\section{References}

1. Pryor J, Akkus E, Alter G, et al. Peyronie's disease. J Sex Med 2004;1:110-5.

2. Grasso M, Lania C, Blanco S, et al. The natural history of Peyronie's disease. Arch Esp Urol 2007;60:326-31.

3. Bilgutay AN, Pastuszak AW. Peyronie's disease: a review of etiology, diagnosis, and management. Curr Sex Health Rep 2015;7:117-31.

4. Yafi FA, Pinsky MR, Sangkum P, et al. Therapeutic advances in the treatment of Peyronie's disease. Andrology 2015;3:650-60.

5. Ralph D, Gonzalez-Cadavid N, Mirone V, et al. The management of Peyronie's disease: evidence-based 2010 guidelines. J Sex Med 2010;7:2359-74.

6. Lin CS, Lin G, Wang Z, et al. Upregulation of monocyte chemoattractant protein 1 and effects of transforming growth factor-beta 1 in Peyronie's disease. Biochem Biophys Res Commun 2002;295:1014-9.

7. Lin GT, Wang Z, Liu BC, et al. Identification of potential biomarkers of Peyronie's disease. Asian J Androl 2005;7:237-43.

8. Wang Z, Lin G, Lue TF, et al. Wogonin suppresses 
cellular proliferation and expression of monocyte chemoattractant protein 1 in Peyronie's plaque-derived cells. BJU Int 2003;92:753-7.

9. Kelâmi A. Autophotography in evaluation of functional penile disorders. Urology 1983;21:628-9.

10. Rosen RC, Riley A, Wagner G, et al. The international index of erectile function (IIEF): a multidimensional scale for assessment of erectile dysfunction. Urology 1997;49:822-30.

11. Paulis G, Barletta D, Turchi P, et al. Efficacy and safety evaluation of pentoxifylline associated with other antioxidants in medical treatment of Peyronie's disease: a case-control study. Res Rep Urol 2015;8:1-10.

12. Levine LA, Larsen SM. Surgery for Peyronie's disease. Asian J Androl 2013;15:27-34.

13. Gholami SS, Lue TF. Correction of penile curvature using the 16-dot plication technique: a review of 132 patients. J Urol 2002;167:2066-9.

14. Lue TF, El-Sakka AI. Venous patch graft for Peyronie's disease. Part I: technique. J Urol 1998;160:2047-9.

15. Zhao T, Tang H, Xie L, et al. Scutellaria baicalensis Georgi. (Lamiaceae): a review of its traditional uses, botany, phytochemistry, pharmacology and toxicology. J Pharm Pharmacol 2019;71:1353-69.

16. Hu Q, Noor M, Wong YF, et al. In vitro anti-fibrotic activities of herbal compounds and herbs. Nephrol Dial Transplant 2009;24:3033-41.

17. Meng XM, Ren GL, Gao L, et al. Anti-fibrotic effect of wogonin in renal tubular epithelial cells via Smad3dependent mechanisms. Eur J Pharmacol 2016;789:134-43.

18. Hu Q, Gao L, Peng B, et al. Baicalin and baicalein attenuate renal fibrosis in vitro via inhibition of the TGFbeta1 signaling pathway. Exp Ther Med 2017;14:3074-80.

19. Kong EK, Yu S, Sanderson JE, et al. A novel anti-fibrotic agent, baicalein, for the treatment of myocardial fibrosis in spontaneously hypertensive rats. Eur J Pharmacol 2011;658:175-81.

20. Pan TL, Wang PW, Leu YL, et al. Inhibitory effects of Scutellaria baicalensis extract on hepatic stellate cells through inducing G2/M cell cycle arrest and activating ERK-dependent apoptosis via Bax and caspase pathway. J Ethnopharmacol 2012;139:829-37.

21. Liu T, Dai W, Li C, et al. Baicalin Alleviates Silica-Induced Lung Inflammation and Fibrosis by Inhibiting the Th17 Response in C57BL/6 Mice. J Nat Prod 2015;78:3049-57.

22. Capoccia E, Levine LA. Contemporary Review of Peyronie's Disease Treatment. Curr Urol Rep 2018;19:51.

23. Li M, Shi A, Pang H, et al. Safety, tolerability, and pharmacokinetics of a single ascending dose of baicalein chewable tablets in healthy subjects. J Ethnopharmacol 2014;156:210-5.

24. Carson CC 3rd, Sadeghi-Nejad H, Tursi JP, et al. Analysis of the clinical safety of intralesional injection of collagenase Clostridium histolyticum $(\mathrm{CCH})$ for adults with Peyronie's disease (PD). BJU Int 2015;116:815-22.

25. Lipshultz LI, Goldstein I, Seftel AD, et al. Clinical efficacy of collagenase Clostridium histolyticum in the treatment of Peyronie's disease by subgroup: results from two large, double-blind, randomized, placebo-controlled, phase III studies. BJU Int 2015;116:650-6.

26. García-Gómez B, González-Padilla DA, Alonso-Isa M, et al. Plication techniques in Peyronie's disease: new developments. Int J Impot Res 2020;32:30-6.

27. Garcia-Gomez B, Ralph D, Levine L, et al. Grafts for Peyronie's disease: a comprehensive review. Andrology 2018;6:117-26.

28. Hatzichristodoulou G. Grafting techniques for Peyronie's disease. Transl Androl Urol 2016;5:334-41.

29. Chang JA, Gholami SS, Lue TF. Surgical management: saphenous vein grafts. Int J Impot Res 2002;14:375-8.

30. Akkus E, Ozkara H, Alici B, et al. Incision and venous patch graft in the surgical treatment of penile curvature in Peyronie's disease. Eur Urol 2001;40:531-6; discussion 537.

31. Hatzimouratidis K, Eardley I, Giuliano F, et al. EAU guidelines on penile curvature. Eur Urol 2012;62:543-52.

32. Seveso M, Melegari S, De Francesco O, et al. Surgical correction of Peyronie's disease via tunica albuginea plication: long-term follow-up. Andrology 2018;6:47-52.

33. Savoca G, Trombetta C, Ciampalini S, et al. Long-term results with Nesbit's procedure as treatment of Peyronie's disease. Int J Impot Res 2000;12:289-93.

34. Levine LA, Greenfield JM. Establishing a standardized evaluation of the man with Peyronie's disease. Int J Impot Res 2003;15 Suppl 5:S103-12.

35. Gur S, Limin M, Hellstrom WJ. Current status and new developments in Peyronie's disease: medical, minimally invasive and surgical treatment options. Expert Opin Pharmacother 2011;12:931-44.

Cite this article as: $\mathrm{Li} \mathrm{WJ}$, Bao J, Zheng DC, Guo J, Xie MK, Chen HY, Wang Z. Treatments of Peyronie's disease with Scutellaria baicalensis and surgery according to the disease course: a single-center retrospective study of 261 patients. Ann Palliat Med 2021;10(3):2979-2989. doi: 10.21037/apm-20-2389 